\title{
Premières notes sur la composition en acides aminés des aliments destinés aux animaux domestiques à Madagascar
}

\author{
par le Pharmacien-Commandant R. GAULIER \\ avec la collaboration technique de MIIe D. CAYRIER
}

\begin{abstract}
RÉSUMÉ
La composition en acides aminés de nombreux aliments produits à Madagascar et destinés localement à l'alimentatıon des animaux domestiques est encore mal connue.

Nous en avons entrepris l'étude d'une foçon systématique par la méthode de MOORE, STEIN et SPACKMAN (chromalographie sur colonne)

Le Tryptophane et la Cystine qui ne figurent pas dans les présents résultats feront l'objet de travaux ultérieurs.

Nous donnons les résultals de nos premières analyses qui ont porté sur :

- les fevilles de Mûrier.

- les graines de Typhonodorum madagascarıensis.

- les tourteaux d'arachides.

- la farine de sang locale.

- la farine de viande ef os lacale.
\end{abstract}

\section{INTRODUCTION}

Le problème de l'alımentatıon protıdıque tıent une place prépondérante dans la nutrition des animaux domestiques.

li est bien évident que la nature de l'apport azoté sera fonction des ressources partıculières à chaque pays, voire même à chaque région.

La composition en acides aminés de nombreux aliments, tels qu'ils sont utilisés en Europe, Amérique etc..., a été établıe, et ces données permettent la préparation de rations équlibrées. Par contre, parmi les produits utilısés de façon courante dans l'alımentation des animaux domestiques à Madagascar, nombreux sont ceux pour lesquels il n'existe que des renseignements fragmentaires quant à leur compositıon.

C'est pourquoi le Laboratorre Central de l'Elevage de Tananarive a inscrit à son programme de recherches l'étude des acides amınés dans les principaux aliments du bétaıl employés à Madagascar.

Ces aliments sont, d'une part, d'origine végétale : graınes de Vigna Sinensis, de Vıha, graines et feuilles de Soja, feuilles de Mûrier, pors du Cap, son et brisures de riz; maïs, drèches de brasserie, tourteaux d'arachides etc...

D'autres sont d'origine animale, tels que : la farine de sang, la farine de viande et os, la farine d'as verts eic.... préparés dans diverses usines de Madagascar, selon des Techniques parfois très différentes des procédés classiques.

\section{MATÉRIEL ET TECHNIQUE}

Pour l'identification et le dosage des acides aminés nous utılisons la méthode Chromatographique sur colonne, décrite par MOORE, SPACKMAN et STEIN, dans plusieurs publications (1) et étudiée par BUSSON, CARBIENER et LANZA (2). 
Cette technique a l'avantage sur les autres méthodes chimiques ou microbiologiques, de détermıner par un chromatogramme unique, l'identification et, avec une très bonne précision ( \pm 5 p. 100), le dosage des 18 principaux aminoacides.

Nous effectuons l'hydrolyse en milieu acide chlorhydrique $6 \mathrm{~N}$, à $135^{\circ} \mathrm{C}$ pendant $24 \mathrm{~h}$.

La chromatographie des Acides aminés acides et neutres s'effectue sur colonne de $150 \mathrm{~cm}$ (diamètre intérieur $9 \mathrm{~mm}$ ) d'Amberlite (IR 120) (fraction $D$ de la séparation hydraulique de Hamilton) avec des tampons de $\mathrm{pH} 3,25$ puis 4,25. La température est maintenue à $50^{\circ} \mathrm{C}$ pendant toute la durée de la Chromatographie. L'effluent est recueilli par fractions de $1 \mathrm{ml}$, sur collecteur ERAL, dans des tubes à essaıs calıbrés.

La Chromatographie des acides aminés basiques est conduite sur colonne de $15 \mathrm{~cm}$ (diamètre intérieur $9 \mathrm{~mm}$ ) d'Amberlite IR 120 (Fraction $\mathrm{C}$ de la séparation hydraulique de Hamilton) avec tampon de $\mathrm{pH} 5,28$. La température est également maintenue à $+50^{\circ} \mathrm{C}$, pendant toute la durée de l'expérience. Les fractions d'effluent $(1 \mathrm{ml})$ sont recueillies sur collecteur SHANDON. La coloration est faite au moyen du Réactif de MOORE et STEIN (3) à la ninhydrine. Les lectures sont faites au photocolorımètre Lumetron et exprimées en équivalents leucine, puis les valeurs trouvées sont transformées en poids des acides aminés respectifs.

Signalons enfin que le tryptophane, détruit au cours de l'hydrolyse acide, ne figure pas dans nos résultats. II en est de même de la cystine dont la déterminatıon nécessite un appareillage spécial dont nous n'avons pas disposé pour nos premières analyses. Des recherches ultérieures nous permettront de compléter ces résultats.

\section{INTERPRÉTATION DES RÉSULTATS}

Pour apprécier la valeur nutritive des protides alımentaires étudiés, nous avons comparé nos résultats avec des formules classiques d'aliments de même type, lorsque nous avons pu trouver ces données dans la littérature (ex : farine de sang, farine de viande et os, tourteaux d'arachides...).

Pour les autres produits (feuilles de mûrier et graines de viha dans le présent article), nous avons comparé les teneurs des différents acides aminés avec celles de l'œuf pris comme étalon de référence, selon la méthode de MITCHELL et BLOCK. Aussi imparfaite que soit I'application de cette méthode dans le cadre de notre étude, puisque les aliments analysés sont destinés à des espèces animales très variées ne demandant pas le même équilibre d'Acides aminés, nous l'avons cependant utilisée en tant que méthode classıque de référence.

Nous donnons cl-après le résultat de nos premières analyses.

\section{LE MURIER}

On trouve le môrier à Madagascar sous des formes très varićes, arbres, arbustes ou buissons. If n'y est habituellement pas cultivé, mais ses feuilles fournissent un fourrage très nutritif, riche en particulier en protides e† en sels minéraux (calcium) et très recherché des animaux.

Nos analyses ont porté sur des feulles de morier provenant d'un arbuste de 6 ans environ, taillé 3 ou 4 fois par an.

Nous avons étudié des feuilles jeunes, âgées de 2 mois, et des feuilles de 5 mois, c'est-à-dire arrivées en fin de croissance.

Leur teneur en Acides aminés figure au tableau II, et elle a été comparée avec celle des protides de l'œuf.

Si nous comparons maintenant la composition des protides des feuilles jeunes avec celle des feuilles adultes, nous constatons que les pourcentages de la plupart des Acides aminés ont varié d'une façon très sensible au cours de la croissance. Cette évolution nous a paru présenter un certain intérêt en nutrition animale, aussi avons nous estimé utıle d'insister sur ce point.:

- la teneur de certains Acides aminés exprimée pour 100 parties de protides a augmenté avec l'âge de la feuille. Ce sont l'acide aspartique (en augmentation de 48 p. 100 par rapport aux feuilles jeunes), la thréonine $(+35$ p. 100), la sérine et la proline $(+16$ p. 100), la méthionine $(+10$ p. 100) et l'histidine ( +7 p. 100):

- par contre, elle est inférieure dans les feuilles en fin de croissance, pour la leucine (-25 p. 100), l'alanine ( -20 p. 100), la tyrosine (-17 p. 100), la glycine (-16 p. 100), 
l'acide glutamique et la lysine (-13 p. 100), l'isoleucine (-11 p. 100), la valine (-9 p. 100) et la phénylalanıne ( -8 p. 100).

\section{Graines de Viha}

Le viha (Typhonodorum madagascariensis) est une monocotylédone de la famille des aracées. C'est une plante herbacée poussant dans les marécages, en particulier ceux de la côte Est de Madagascar.

Ses granes sont utilisées dans l'alimentation du bétail.

Nos analyses ont porté sur des graines non décortiquées. Leur composition biachimique figure au tableau I.

Nous avons donné dans le tableau II leur composition en acides aminés que nous avons comparée avec les protides de l'œuf.

\section{Tourteaux d'arachides}

Sous-produit de l'industrie oléagineuse, les tourtecux d'arachides représentent dans l'alimentation animale à Madagascar, la principale source de protides d'origine végétale.

Ce sont des tourteaux de pression, aussi leur teneur en matières grasses est-elle relativement élevée ( 8 à 10 p. 100, avec des écarts parfois importants), ce qui n'est pas sans inconvénient pour leur conservation. Ils sont généralement obtenus par le procédé de pression contınue (Expeiler) et à chaud ( 90 à $110^{\circ} \mathrm{C}$ ), ce qui leur confère une teinte brune plus ou moins accentuée (4).

Nous avons analysé 2 échantillons.

Le tourteau No 1 provient du traitement de graines d'orachides spécialement cultivées pour l'huilerıe.

Le tourteau No 2 provient du traitement des graines constituant les déchets de triage pour arachides de bouche.

De la comparaison de la composition des protides de ces 2 tourteaux (cf Tableau III), on constate des différences sensibles dans les teneurs de certains acides aminés :

Le tourteau No 1 est plus riche que l'échantillon No 2, en histidine $(+31$ p. 100), prolıne $(+30$ p. $100)$, tysine $(+17$ p. 100), tyrosine $(+14$ p. 100), valine $(+13$ p. 100$)$.
II est moins riche en thréonine ( -13 p. 100) et en sérine ( -12 p. 100).

En comparant enfin les 2 produits locaux avec le fourteau classique, on constate que ceux-là on une teneur supérieure en méthıonine et en isoleucine, mais qu'ils sont moins riches en valine, leucine et phénylalanine que le produit classique.

\section{Farine de Sang}

Ce produit est, dans une usine de Tananarive, préparé essentıellement à partır de sang de bouf, accessoirement mélangé avec du sang de porc. Le sang est parfols privé au préalable d'une partie de son plasma, destıné à d'autres usages (conserverie).

Le sang, coagulé, est chauffé pendant 5 h à une température voisine de $80^{\circ} \mathrm{C}$, avec brassage continu. Au cours de cette opération, le produit perd environ 30 p. 100 d'eau.

La masse obtenue est ensuite étalée sur une aıre cımentée où elle subit un pré-séchage au soleil. Pendant cette opération, elle est brassée périodiquement, et réduite progressivement en une poudre granuleuse. La dessiccation est ensulte poursuivie sous hangar ventılé.

L'utılisateur doit généralement procéder à un broyage du produit avant de l'incorporer dans les rations alimentares.

La composition de la farine de sang en Acides aminés figure au Tableau III. Elle diffère de la farine classique par une teneur plus élevée en Méthıonine. Par contre, les taux de glycine, de lysine, et surtout d'isoleucine sont nettement inférieurs.

Les autres Acides amınés comparés sont sensiblement équivalents.

\section{Farine de viande et os}

A notre connalssance une seule usine, installée dans la région de Diégo-Suarez, procède à la fabrication de ce produit à Madagascar.

Les matières premières utilisées comprennent: pour 50 p. 100 les os provenant de la carcasse entière de l'anımal, pour 45 p. 100 des viscères, et pour 5 p. 100 des déchets et saisies.

La méthode de préparation donne lieu aux opérations suivantes :

- Broyage mécanique des os: 
TABLEAU No 1

Composition chimique (1)

(pour 100 de produit brut)

\begin{tabular}{|c|c|c|c|c|c|c|c|}
\hline & $\begin{array}{l}\text { Feuilles de } \\
\text { Marier jeunes }\end{array}$ & $\begin{array}{l}\text { Fevilles de } \\
\text { inturier en fin } \\
\text { de croissance }\end{array}$ & $\begin{array}{l}\text { Graines } \\
\text { de Viha }\end{array}$ & $\begin{array}{c}\text { Tourteau } \\
\text { d'Arachide } \\
\text { No } 1\end{array}$ & $\begin{array}{c}\text { Tourteau } \\
\text { d'Arachide } \\
\text { No } 2\end{array}$ & $\begin{array}{l}\text { Farine } \\
\text { de Sang }\end{array}$ & $\begin{array}{l}\text { Farine de } \\
\text { Viande et Os }\end{array}$ \\
\hline Eau & 70,20 & 65,61 & 65,06 & 8,12 & 10,74 & 17,77 & 3,75 \\
\hline Matières minérales & 3,00 & 5,76 & 1,56 & 6,69 & 6,29 & 4,93 & 34,62 \\
\hline Matières grasses & 1,15 & 1,62 & 0,41 & 8,11 & 7,72 & 0,66 & 11,18 \\
\hline $\begin{array}{l}\text { Matières azotées } \\
(\mathrm{N} \times 6,25)\end{array}$ & 7,49 & 6,46 & 3,88 & 45,13 & 47,59 & 71,77 & 49,40 \\
\hline Cellulose brute & 2,19 & 3,45 & 1,44 & 4,60 & 5,19 & - & - \\
\hline Extractif non azoté & 15,97 & 17,10 & 27,65 & 27,35 & 22,47 & 4,87 & 1,05 \\
\hline
\end{tabular}

(1) Ies techntques utilisées sont celles décrites dans la Miise à Jour au 1er Juin 1961 des méthodes officielles employées par les Laboratoires du Service de la Répresston des Fraudes pour l'analyse des échantillons de prodicits de l'alimentation animale" Laboratoire d'Alimentation de l'Ecole Nationale Vétérinaire d'Alfort.

Fascicule XXVI, BOSC Frères, EdAteur, Iyon. 1961. 


\begin{tabular}{|c|c|c|c|c|c|c|c|c|c|}
\hline & \multicolumn{3}{|c|}{ Peuilles de vitrzer jeures } & \multicolumn{3}{|c|}{ Fevilles de Wurier en tin de croissance } & \multicolumn{3}{|c|}{ Graines de viha } \\
\hline & $\begin{array}{c}\text { Pour } 100 \\
\text { de Produit } \\
\text { Sec }\end{array}$ & $\begin{array}{l}\text { Pour } 100 \\
\text { de } \\
\text { Protidea }\end{array}$ & $\begin{array}{c}\text { Différences avec } \\
\text { les Protides } \\
\text { totaux } \\
\text { de l'oeuf }(1)\end{array}$ & $\begin{array}{c}\text { Pour 100 } \\
\text { de Produit } \\
\text { Sec }\end{array}$ & $\begin{array}{l}\text { Pour } 100 \\
\text { de } \\
\text { Protides }\end{array}$ & $\begin{array}{c}\text { Différences avec } \\
\text { les Protides } \\
\text { totaux } \\
\text { de l'oeuf }(1)\end{array}$ & $\begin{array}{l}\text { Pour } 100 \\
\text { de Produit } \\
\text { Sec }\end{array}$ & $\begin{array}{c}\text { Pour } 100 \\
\text { de } \\
\text { Protides }\end{array}$ & $\begin{array}{c}\text { Différence avec } \\
\text { les Protides } \\
\text { totaux } \\
\text { de l'oeuf (1) }\end{array}$ \\
\hline Aciòe Aspartique & 1,61 & 6,40 & -22 & 1,77 & 9,44 & +15 & 1,25 & 11,22 & +37 \\
\hline Threonine & 0,71 & 2,82 & -42 & 0,71 & 3,80 & -22 & 0,55 & 4,98 & +2 \\
\hline Sérine & 0,69 & 2,76 & -65 & 0,60 & 3,20 & -59 & 0,71 & 6,40 & -18 \\
\hline Acide Gutanique & 2,20 & 8,74 & -31 & 1,43 & 7,62 & -40 & 1,00 & 8,98 & -29 \\
\hline Proline & 1,11 & 4,42 & -2 & 0,97 & 5,14 & +14 & 0,48 & 4,34 & -4 \\
\hline Glycine & 1,40 & 5.58 & +132 & 0,38 & 4,58 & +95 & 0,45 & $4, \infty 8$ & +70 \\
\hline Alanine & 1,63 & 6.50 & & 0,98 & 5,20 & & 0,41 & 3,68 & \\
\hline Valine & 1,43 & 5,70 & -22 & 0,98 & 5,20 & -29 & 0,60 & 5,42 & -26 \\
\hline Méthionine & 0.46 & 1,82 & -56 & 0,38 & 2,00 & -51 & 0,23 & 2,04 & -50 \\
\hline Isoloucine & 1,15 & 4,56 & -43 & 0,76 & 4,06 & -49 & 0,29 & 2,58 & -68 \\
\hline Leucine & 2,19 & 8,72 & -5 & 1,23 & 6,54 & -29 & 0,71 & 6,42 & -30 \\
\hline Tyrosine & $i, 02$ & 4,04 & -10 & 0,63 & 3,36 & -25 & 0,40 & 3,58 & -20 \\
\hline Phényl-alanine & 1,39 & 5,52 & -12 & 0,96 & 5,08 & -19 & 0,45 & 4,02 & -36 \\
\hline Lysine & 1,06 & 4,20 & -42 & 0,69 & 3,66 & -49 & 0,48 & 4,28 & -41 \\
\hline Histidine & 0,53 & 2,10 & 0 & 0,42 & 2,24 & +7 & 0,25 & 2,28 & +9 \\
\hline Arginine & $t, 45$ & 5,78 & -10 & 1,07 & 5,70 & -11 & 0.99 & 8,94 & +40 \\
\hline
\end{tabular}

(1) Exprimées en pour 100 pour chaque acide aminé, sur la base de $16 \mathrm{~g}$. d'Azote. 
Composition en acides aninés

\begin{tabular}{|c|c|c|c|c|c|c|c|c|c|c|c|}
\hline & \multicolumn{2}{|c|}{$\begin{array}{c}\text { Tourteau d Arachide } \\
\text { local No } 1\end{array}$} & \multirow{2}{*}{\begin{tabular}{|c|}
$\begin{array}{c}\text { Tourteau } \\
\text { d'Arachide } \\
\text { classique (1) }\end{array}$ \\
$\begin{array}{c}\text { Pour } 100 \\
\text { de Produit } \\
\text { sec }\end{array}$ \\
\end{tabular}} & \multicolumn{2}{|c|}{$\begin{array}{c}\text { Tourteau d'A rachide } \\
\text { Local NO2 }\end{array}$} & \multicolumn{2}{|c|}{$\begin{array}{c}\text { Farine de Sang } \\
\text { locale }\end{array}$} & \multirow{2}{*}{$\begin{array}{c}\begin{array}{c}\text { Farine } \\
\text { de Sang } \\
\text { classique(1) }\end{array} \\
\begin{array}{c}\text { Pour } 100 \\
\text { de Produit } \\
\text { sec }\end{array}\end{array}$} & \multicolumn{2}{|c|}{$\begin{array}{l}\text { Farine de Viande } \\
\text { et os locile }\end{array}$} & \multirow{2}{*}{$\begin{array}{l}\begin{array}{l}\text { Farine de } \\
\text { viande à } \\
50 p \cdot 100 \\
\text { classique (1) }\end{array} \\
\begin{array}{c}\text { Pour 100 } \\
\text { de Produjt } \\
\text { sec }\end{array}\end{array}$} \\
\hline- & $\begin{array}{l}\text { Pour } 100 \\
\text { de Produit } \\
\text { seo }\end{array}$ & $\begin{array}{c}\text { Pour } 100 \\
\text { de } \\
\text { Protides }\end{array}$ & & $\begin{array}{c}\text { Pour 100 } \\
\text { de Produit } \\
\text { sec }\end{array}$ & $\begin{array}{l}\text { Pour } 100 \\
\text { de } \\
\text { Protides }\end{array}$ & $\begin{array}{c}\text { Pour } 100 \\
\text { de Produit } \\
\text { sec }\end{array}$ & $\begin{array}{l}\text { Pour } 100 \\
\text { de } \\
\text { Protides }\end{array}$ & & $\begin{array}{c}\text { Pour } 100 \\
\text { de Produit } \\
\text { sec }\end{array}$ & $\begin{array}{l}\text { Pour } 100 \\
\text { de } \\
\text { Protides }\end{array}$ & \\
\hline Acido Aspartique & 5,13 & 10,44 & & 6,01 & 11,28 & 8,85 & 10,13 & & 3,15 & 6,14 & \\
\hline Thréonino & 1,21 & 2,46 & 1,20 & 1,48 & 2,78 & 4,17 & 4,77 & 4,09 & 1,38 & 2,68 & 2,10 \\
\hline Sérine & 2,22 & 4,52 & & 2,69 & 5,04 & 4,70 & 5,38 & & 1,85 & 3,60 & \\
\hline Acide Glutamique & 7,43 & 15,14 & & 8,56 & 16,06 & 6,94 & 7,94 & & 4,96 & 9,66 & \\
\hline Proline & 2,65 & 5,40 & & 2,03 & 3,80 & 3,18 & 3,64 & & 3,73 & 7,26 & \\
\hline Glycine & 2,52 & 5,14 & 2,70 & 2,70 & 5,06 & 3,45 & 3,95 & 5,00 & 6,89 & 13,42 & 2,00 \\
\hline Alanine & 1,75 & 3,56 & & 1,97 & 3,70 & 7,20 & 8,25 & & 4,05 & 7,90 & \\
\hline Valine & 2,20 & 4,48 & 2,74 & 2,07 & 3,88 & 8,13 & 9,31 & 7,79 & $1,84$. & 3,58 & 2,70 \\
\hline Méthionine & 0,72 & 1,46 & 0,50 & 0,81 & 1,52 & 1,87 & 2,14 & 1,12 & 0,80 & 1,56 & 0,70 \\
\hline Isoleucine & 1,70 & 3,46 & 1,50 & 1,79 & 3,36 & 0,68 & 0,78 & 1,21 & 1,09 & 2,12 & 2,30 \\
\hline Leucine & 3,13 & 6,38 & 3,50 & 3,32 & 6,22 & 10,67 & 12,22 & 11,50 & 2,45 & 4,78 & 4,70 \\
\hline Tyrosine & 2,02 & 4,12 & 2,00 & 1,89 & 3,55 & 2,13 & 2,44 & 1,96 & 0,87 & 1,70 & 1.70 \\
\hline Phényl-alanine & 2,45 & 4,98 & 2,70 & 2,51 & 4.70 & 5.89 & 6,75 & 6,01 & 1,53 & 2,98 & 2,40 \\
\hline Lysine & 1,75 & 3.56 & 1,50 & 1,58 & 2,96 & 7,62 & 8,72 & 9,17 & 2,33 & 4,54 & 2,70 \\
\hline Hrstidjne & 1,17 & 2.38 & 0,93 & 0,87 & 1,64 & 4,87 & 5.57 & 5,03 & 0,70 & 1,36 & 1,40 \\
\hline Arginine & 5,47 & 11,14 & 5,50 & 5,48 & 10,28 & 3,47 & 3,97 & 3,59 & 3,53 & 6,88 & 3,00 \\
\hline
\end{tabular}

(1) Réf : Jacquot, Le Bars et Simonnet : Niutrition Animale (1958) Vol. I page 176-177 
- Cuisson et dessiccation pendant $8 \mathrm{~h} \mathrm{ò} 130^{\circ} \mathrm{C}$, de l'ensemble des matières premıères. Les lipides sont partiellement élıminés par centrifugation ;

- Broyage de la matière sèche :

- Mise en sacs en nylon à la sortie du broyeur. par poids standard de $50 \mathrm{~kg} \mathrm{(1).}$

Le produit terminé analysé a une teneur en protides totaux de 51,32 p. 100 de matière sèche, donc vasine de celle d'une farine de viande d 50 p. 100. Cependant, la composition en Acides aminés de la Farine de viande et os figurant au tableau III montre que ce produit diffère très nettement de la composition d'une farme de viande à 50 p. 100 classique.

(1) Les renseignements concernant celte fabrication nous ont éré communıqués par la Délégation Provinciale à l'Elevage de Diégo-Suarez.
Le taux de glycine est considérablement plus élevé dans la farine locale. Ce fait est dû à l'utilisation d'un pourcentage très important de tissus osseux et viscéraux, riches en gélatine, dans la préparation du produit local.

Les teneurs en méthionıne et arginine sont satisfassantes. Par contre les taux de tous les autres Acides aminés comparés sont nettement inférieurs à ceux de la farine classique (47 p. 100 pour l'isoleucine, 50 p. 100 pour l'histidine, 51 p. 100 pour la tyrosine, 52 p. 100 pour la leucıne, 64 p. 100 pour la phénylalanıne, 66 p. 100 pour la thréonine).

Institut d'Elevage et de Médecine Vétérinare des Pays Tropicoux

Laboratorre Central de l'Elevage de Tonanarive

\section{SUMMARY}

\section{Preliminary notes on the amino acid composition of food materials destined for domestic animals in Madagascar}

The amino acid composition of numerous food materials produced in Madagascar and destined to feed the local domestic animals is still barely known.

We have undertaken their systematic study using the method according to MOORE, STEIN, SPACKMAN (column chromatography)

Tryptophan and Cyslıne that are not listed with the present results, will form the subject of further study later on.

The results are given of our first series of analyses which were carried out on :

- Mulberry leaves.

- The seeds of Typhonodorum madagoscarrensis.

- Ground - nut oil - cake.

- Local « blood» meal.

- Local meat and bone meal.

\section{RESUMEN}

\section{Primeras notas sobre la composición en ácidos aminados de los} alimentos para los animales domésticos en Madagascar

Se conoce todavia mal la composıción de âcidos aminados de nûmerososalımentos producidos en Madagascar y destinados localmente a la alımentación de los animales domésticos.

El estudio se hace de un modo sistemàtico mediante el método MOORE, STEIN y SPACKMAN (cromatografia en columna).

El triptofono y la cistina no figuran en los presentes resultados, fueron el objeto de trabajos ulteriores

Se dan los resultados de los primeras análisis que traiaron de :

- las hojas de morera .

- los granos de typhanodorum modagascariensis.

- las torlas de cacahuete.

- la harina de sangre local.

- la harina de carne y hueso local. 


\section{BIBLIOGRAPHIE}

1. MOORE (S.) et STEIN (W. H.). - Chromatography of Amino-acids on sulfonated polystyrene resins. The journal of Biological Chemistry. 1951, 192, 663-681.

MOORE (S.), SPACKMAN (D.) et STEIN (W. H.). - Anolytical Chemistry, 1958, 30, 1185 et sq.

2. BUSSON (F.), CARBIENER (R.) et LANZA (J.). - Méthodes chromatographiques de dosage des Acides aminés. Les Cahiers Techniques du Centre de Coordination des Etudes ef
Recherches sur lo Nutrition ef l'Alimentation V-CNRS, 1960.

3. MOORE (S.) et STEIN (W. H.). - A modified ninhydrin reagent for the photometric determination of amino-acids and related compounds. The journal of Biological Chemistry, 1954, 211, 907-913.

4. DAUMAS (R.). - Technologie et composition des tourteaux de Madagascar. Revue d'Elevage et de Médecine Vétérinaire des Pays Tropicoux, 1963, 2, 237-261. 